\title{
On the RF Properties of Weakly Saturated SiGe HBTs and Their Potential Use in Ultralow-Voltage Circuits
}

\author{
Sachin Seth, Student Member, IEEE, Laleh Najafizadeh, Member, IEEE, and John D. Cressler, Fellow, IEEE
}

\begin{abstract}
We investigate, for the first time, the feasibility of operating silicon-germanium (SiGe) heterojunction bipolar transistors (HBTs) in a weakly saturated bias regime to enable ultralow-voltage RF front-end design. Measured $d c, a c$, and RF characteristics of third-generation high-performance SiGe HBTs operating in weak saturation are presented. Robust RF operation of $0.12 \times 6.0 \mathrm{\mu m}^{2} \mathrm{SiGe}$ HBTs are demonstrated in a common-emitter configuration at collector-to-emitter voltages above $0.15 \mathrm{~V}$. A noise figure of $1.33 \mathrm{~dB}$ and an input third-order intercept point above $-8 \mathrm{dBm}$ for a $3-\mathrm{GHz}$ input tone are achieved at $0.30 \mathrm{~V}$. These results have potential implications for RF circuits used in severely power-constrained systems.
\end{abstract}

Index Terms-Biomedical telemetry, heterojunction bipolar transistors (HBTs), low-voltage operation, RF circuit design, silicon-germanium (SiGe), SiGe HBTs.

\section{INTRODUCTION}

$\mathbf{S}$ ILICON-GERMANIUM ( $\mathrm{SiGe}$ ) is making inroads in a wide variety of mixed-signal circuit applications due to its attractive combination of excellent RF performance metrics at conservative lithographic feature size, together with high integration levels and yield associated with standard silicon manufacturing. With peak operational frequencies rapidly pushing toward $500 \mathrm{GHz}$ (at $130 \mathrm{~nm}$ !), SiGe heterojunction bipolar transistors (HBTs) are inevitably associated with very high performance circuit designs [1]. For severely power-constrained wireless systems, such as biomedical electronics, wireless devices, or other battery-biased systems, however, the inherent high performance of SiGe HBTs can instead be potentially traded off for a lower operating bias currents and, hence, lower power dissipation [2]. In addition to this appealing performance bias-current (e.g., $f_{T} / f_{\max }$ versus $I_{C}$ ) tradeoff, many emerging applications also save power by dramatically lowering the operating voltage, and new circuit-design approaches within the CMOS community have been developed to address this need [3]. One would intuitively assume that low-voltage operation

Manuscript received August 27, 2010; accepted September 26, 2010. Date of publication November 18, 2010; date of current version December 27, 2010. The review of this letter was arranged by Editor M. Ostling.

S. Seth and J. D. Cressler are with the Georgia Institute of Technology, Atlanta, GA 30332 USA (e-mail: sseth3@mail.gatech.edu; cressler@ ece.gatech.edu).

L. Najafizadeh is with the National Institute of Health, Gaithersburg, MD 80523 USA (e-mail: najafizadehl@ mail.nih.gov).

Color versions of one or more of the figures in this letter are available online at http://ieeexplore.ieee.org.

Digital Object Identifier 10.1109/LED.2010.2087313 in SiGe HBTs is not a viable approach since lowering the collector-to-emitter voltage $\left(V_{\mathrm{CE}}\right)$ forces the bipolar transistor into saturation (e.g., if $V_{\mathrm{BE}}=0.9 \mathrm{~V}$ and $V_{\mathrm{CE}}=0.2 \mathrm{~V}$, the collector-base junction is forward biased by $0.7 \mathrm{~V}$ ). Saturating a bipolar transistor floods the base region with excess minoritycarrier charge, severely degrading both $d c$ and $a c$ performance. Therefore, biasing the transistor in its saturation region is widely considered a very bad idea (e.g., circuit families such as CML/ECL were invented to prevent saturation in high-speed logic). Given that modern SiGe HBTs have "performance to burn," a logical question presents itself. For power-constrained low-frequency (e.g., $<5 \mathrm{GHz}$ ) circuits, is it possible to use the SiGe HBT in weak saturation without overly compromising its RF performance metrics while enabling device operation down to very low voltages (e.g., $<0.5 \mathrm{~V})$ and, hence, power? Given that SiGe HBTs enjoy RF-relevant advantages over CMOS at fixed-scaling node (noise figure, $1 / f$ noise, output conductance $g_{m}$ per unit area, matching, etc.), a SiGe HBT operating in weak saturation might offer performance advantages over CMOS solutions at fixed (and highly constrained) power levels. This letter addresses this question for the first time, by measuring the $d c, a c$, noise, and linearity characteristics of weakly saturated SiGe HBTs.

The SiGe HBTs used in this study are from a commercially available third-generation 130-nm SiGe BiCMOS platform [4] and were $0.12 \times 6.0 \mu \mathrm{m}^{2}$ in geometry.

\section{Measurement Results}

\section{A. DC and AC Measurements}

Fig. 1 shows the output characteristics and the current gain $(\beta)$ versus bias current of the SiGe HBT in weak saturation. With both the E-B and the $\mathrm{C}-\mathrm{B}$ junctions forward biased, a collector current greater than $10 \mathrm{~mA}$ is still achieved, which is more than sufficient to bias the device at peak $f_{T}$, while the peak $\beta$ remains above 400 in weak saturation.

On-wafer high-frequency measurements were performed, and pad parasitics were deembedded at every frequency using the traditional open-short method [5]. The peak $f_{T}$ and $f_{\mathrm{MAX}}$ of the device were found to be above $125 \mathrm{GHz}$ at $V_{\mathrm{CE}}=$ $0.30 \mathrm{~V}$ and above $50 \mathrm{GHz}$ at $0.15 \mathrm{~V}$ (Fig. 2), which are more than the acceptable performance for many RF designs. To obtain $f_{T} / f_{\mathrm{MAX}}$, measured $S$-parameters were converted to $H$-parameters/Mason Unilateral Gain, both of which showed classical $20-\mathrm{dB} / \mathrm{dec}$ rolloff with frequency into saturation. 


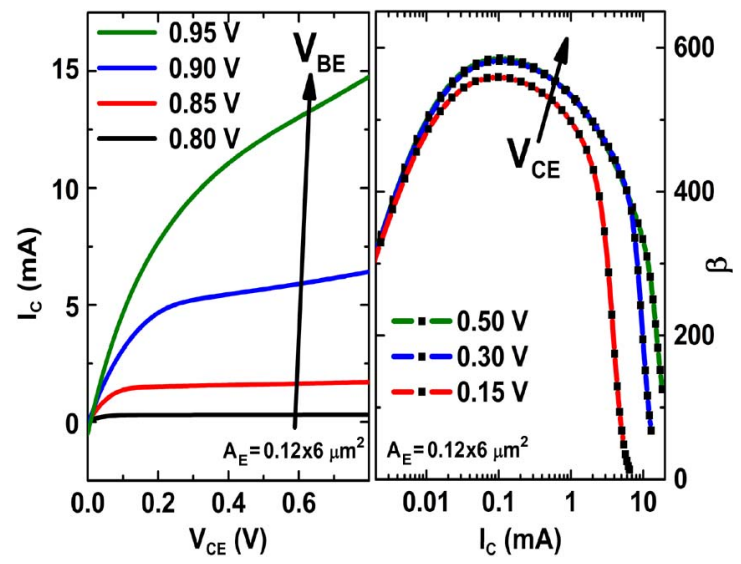

Fig. 1. (Left) Measured forced- $V_{\mathrm{BE}}$ output characteristics of a $0.12 \times$ $6.0 \mu \mathrm{m}^{2}$ SiGe HBT. (Right) Measured current gain $(\beta)$ versus $I_{C}$ of a $0.12 \times 6.0 \mu \mathrm{m}^{2} \mathrm{SiGe}$ HBT for three different $V_{\mathrm{CE}} \mathrm{S}$.

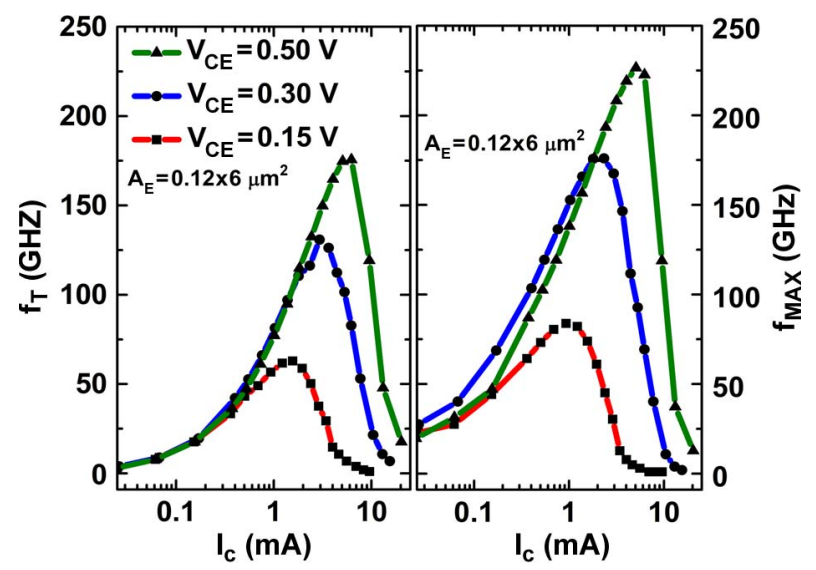

Fig. 2. Measured $f_{T}$ and $f_{\max }$ characteristics versus $I_{C}$ of a $0.12 \times 6 \mu \mathrm{m}^{2}$ SiGe HBT taken at three different $V_{\mathrm{CE}}$ 's.

\section{B. Noise and Linearity Measurements}

1) Power Gain and Linearity (IIP3): Power gain and small-signal linearity [input third-order intercept point (IIP3)] measurements were performed on the saturated SiGe HBT, operating in a common-emitter configuration, and terminated with $50-\Omega$ load and source impedances. The measurement setup is described in [6]. For linearity, the RF power was swept for two input tones (3.000 and $3.008 \mathrm{GHz}$ ), ensuring smallsignal operation, and the output fundamental and third-order intermodulation (IMD) terms were measured. The third-order IMD data obeyed an ideal $3: 1$ slope, allowing extraction of the IIP3 [2].

Fig. 3 shows measured IIP3 results, which reach a peak above -8 and $-10 \mathrm{dBm}$ at a $V_{\mathrm{CE}}$ of 0.30 and $0.15 \mathrm{~V}$, respectively. With increasing $V_{\mathrm{CE}}$, the peak IIP3 increased, consistent with results reported in [7]. The "sweet spot" for high linearity in the IIP3 curves can be exploited by designers to ensure high receiver sensitivity while still biased at low voltages.

Fig. 3 also shows the RF power gain at $3.0 \mathrm{GHz}$. A power gain above $7 \mathrm{~dB}$ at $V_{\mathrm{CE}}=0.30 \mathrm{~V}$ is achieved in weak saturation, while at $0.15 \mathrm{~V}$, the power gain is effectively zero, rendering the device useless. As with linearity, power gain

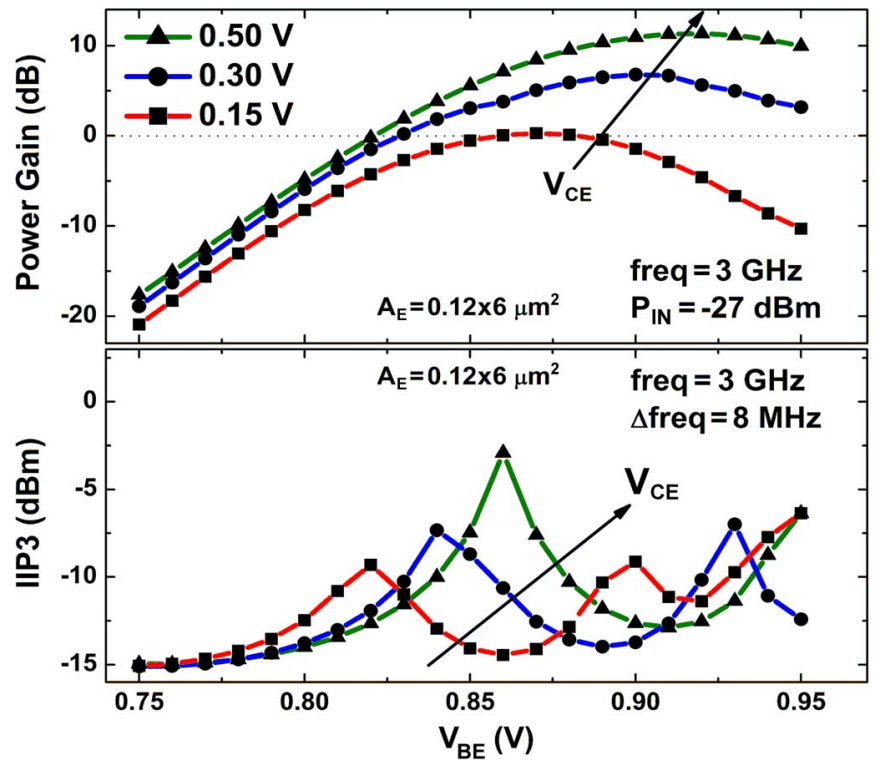

Fig. 3. (Top) Measured power gain versus $V_{\mathrm{BE}}$ for a $0.12 \times 6 \mu \mathrm{m}^{2} \mathrm{SiGe}$ HBT for 3-GHz input tone. (Bottom) Two-tone response of a $0.12 \times 6 \mu \mathrm{m}^{2}$ SiGe HBT at 3-GHz input tone with 8-MHz offset.

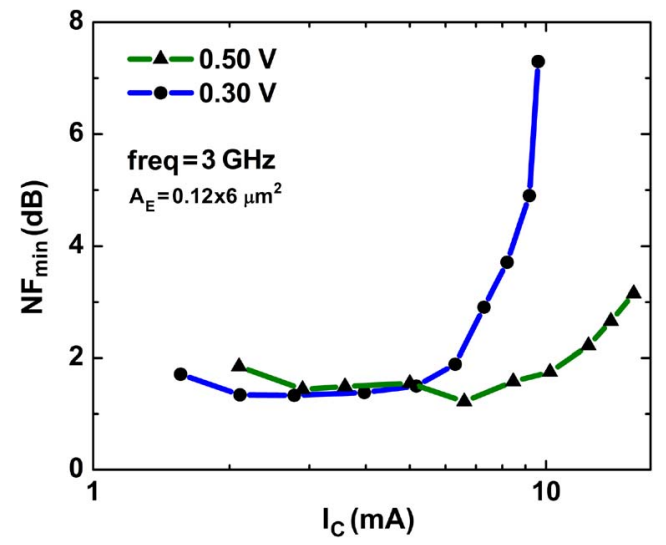

Fig. 4. Measured minimum noise figure $\left(\mathrm{NF}_{\min }\right)$ across bias for $0.12 \times$ $6 \mu \mathrm{m}^{2} \mathrm{SiGe} \mathrm{HBT}$ at different $V_{\mathrm{CE}} \mathrm{s}$.

can be further enhanced using classical approaches, such as cascading and source/load impedance matching.

2) Noise Figure: Noise performance is clearly a key for most RF applications, and here, the noise was characterized in a load-pull setup, with the source impedance varied to find the optimum source impedance $Z_{s \text {,opt }}$ for minimum noise figure $\left(\mathrm{NF}_{\min }\right)$ across bias at $3.0 \mathrm{GHz}$. Fig. 4 shows the minimum noise figure $\left(\mathrm{NF}_{\min }\right)$ across bias for different $V_{\mathrm{CE}}$ values. Sub2-dB NF$F_{\text {min }}$ can easily be achieved under weak saturation.

The impedance where the noise figure is minimum $\left(Z_{s, \mathrm{opt}}\right)$ usually lies in the region between 150 and $200 \Omega$, thus making impedance matching using lumped $L C$ components very easy for RF design. It can also be seen in Fig. 4 that $\mathrm{NF}_{\text {min }}$ is sub- $2 \mathrm{~dB}$ across a wide bias range, allowing dc biasing of HBTs for simultaneous low noise and high-gain performance.

\section{Discussion AND SUMmary}

These results strongly suggest that SiGe HBTs operated in weak saturation represent a potentially viable bias regime for 
TABLE I

Performance Metrics for the SiGe HBt and the Si nFeTs (at the Same Geometry and the Same Power Density). All RF and Noise Parameters Are Measured at $3 \mathrm{GHz}$

\begin{tabular}{|l|c|c|c|c|c|c|}
\hline & \multicolumn{2}{|c|}{ HBT $\left(0.12 \times 6 \mathrm{~m}^{2}\right)$} & \multicolumn{2}{c|}{ nFET $\left(0.12 \times 10 \mu \mathrm{m}^{2}\right)$} & \multicolumn{2}{c|}{ nFET $\left(0.12 \times 1 \times 32 \mu \mathrm{m}^{2}\right)$} \\
\cline { 2 - 7 } & $\mathbf{V}_{\mathbf{C E}}=\mathbf{0 . 5} \mathbf{V}$ & $\mathbf{V}_{\mathbf{C E}}=\mathbf{0 . 3} \mathbf{V}$ & $\mathbf{V}_{\mathbf{D S}}=\mathbf{0 . 5} \mathbf{V}$ & $\mathbf{V}_{\mathbf{D S}}=\mathbf{0 . 3} \mathbf{V}$ & $\mathbf{V}_{\text {DS }}=\mathbf{0 . 5} \mathbf{~ V}$ & $\mathbf{V}_{\mathbf{D S}}=\mathbf{0 . 3} \mathbf{~ V}$ \\
\hline Peak f $(\mathrm{GHz})$ & 175 & 130 & 59 & 48 & 66 & 39 \\
\hline Peak f & & 177 & 80 & 68 & 105 & 41 \\
\hline Peak power gain (dB) & 11 & 6 & -6 & -8 & 4 & 1 \\
\hline Peak IIP3 (dBm) & -3 & -8 & 7 & 5 & 5 & 4 \\
\hline $\mathrm{NF}_{\min }(\mathrm{dB})$ & 1.22 & 1.33 & 2.29 & 2.39 & 1.36 & 1.5 \\
\hline
\end{tabular}

certain power-constrained circuits, particularly for those that are intended for small-signal operation at very low voltages (e.g., LNAs). For an instructive comparison, we measured the RF performance of 130-nm nFETs, both of almost identical size $(W / L=10.0 \mu \mathrm{m} / 130 \mathrm{~nm})$ and identical current-drive capability $(W / L=32.0 \mu \mathrm{m} / 130 \mathrm{~nm})$ to the SiGe HBT used in this study. The results of this comparison are summarized in Table I. It is clearly seen that, for all metrics of comparison (except IIP3), the SiGe HBT outperforms the MOSFET. The FETs are less nonlinear than the HBTs (hence, higher IIP3) due to their square-law $I-V$ relationship, as compared with an exponential $I-V$ relationship in the HBTs. However, the excellent power gain, noise figure, and ease of input impedance matching of the SiGe HBTs more than make up for their reduced IIP3.

One might logically wonder why one can successfully operate advanced SiGe HBTs in weak saturation. We have recently shown [8] that technology scaling naturally improves the inverse-mode operation of SiGe HBTs, a bias regime which also has been historically avoided due to poor performance. In that case, the favorable reductions in the ratio between intrinsic to extrinsic junctions and naturally higher doping in the collector-base junction, as well as the reduced collectorepitaxy thickness, all combine to boost inverse mode $f_{T}$ 's to above $100 \mathrm{GHz}$ using easy optimization steps. Given that the collector-base junction is in play with weakly saturated devices, these facts, together with the natural barrier to forwardbiased collector-base junction charge storage that results from the band offset of the Ge profile itself (here $25 \%$ peak $\mathrm{Ge}$ ), combine to produce the observed impressive performance in weak saturation.

Ultralow-voltage SiGe HBT RF circuits exploiting these results are presently being developed and will be published shortly.

\section{ACKNOWLEDGMENT}

The authors would like to thank the SiGe Team at Georgia Tech for their support.

\section{REFERENCES}

[1] J. D. Cressler, Ed., Silicon Heterostructure Handbook—Materials, Fabrication, Devices, Circuits, and Applications of SiGe and Si Strained Layer Epitaxy. Boca Raton, FL: CRC Press, 2006.

[2] J. D. Cressler and G. Niu, Silicon-Germanium Heterojunction Bipolar Transistors. Norwood, MA: Artech House, 2003.

[3] R. Sarpeshkar, Ultra Low Power Bioelectronics. Cambridge, U.K.: Cambridge Univ. Press, 2010.

[4] B. Jagannathan, M. Khater, F. Pagette, J.-S. Rieh, D. Angell, H. Chen, J. Florkey, F. Golan, D. R. Greenberg, R. Groves, S. J. Jeng, J. Johnson, E. Mengistu, K. T. Schonenberg, C. M. Schnabel, P. Smith, A. Stricker, D. Ahlgren, G. Freeman, K. Stein, and S. Subbanna, "Self-aligned SiGe NPN transistors with $285 \mathrm{GHz} \mathrm{f}_{\mathrm{MAX}}$ and $207 \mathrm{GHz} \mathrm{f}_{T}$ in a manufacturable technology," IEEE Electron Device Lett., vol. 23, no. 5, pp. 258-260, May 2002.

[5] M. Koolen, J. A. M. Geelen, and M. P. J. G. Versleijen, "An improved de-embedding technique for on-wafer high frequency characterization," in Proc. BCTM Symp., 1991, pp. 188-191.

[6] A. Madan, T. Thrivikraman, S. Seth, R. Verma, J. Poh, and J. D. Cressler, "A new and simple measurement approach for characterizing intermodulation distortion without using a spectrum analyzer," in Proc. IEEE SiRF, Jan. 2010, pp. 88-91.

[7] H. F. F. Jos, "A model for the non-linear base-collector depletion layer charge and its influence on intermodulation distortion in bipolar transistors," Solid State Electron., vol. 33, no. 7, pp. 907-915, Jul. 1990.

[8] A. Appaswamy, M. Bellini, W.-M. L. Kuo, P. Cheng, J. Yuan, C. Zhu, J. D. Cressler, G. Niu, and A. J. Joseph, "Impact of scaling on inverse mode operation of SiGe HBTs," IEEE Trans. Electron Devices, vol. 54, no. 6, pp. 1492-1501, Jun. 2007. 